\title{
Hybrid models of solar wind plasma heating
}

\author{
L. Ofman ${ }^{1,2, *}$, A.-F. Viñas ${ }^{2}$, and P. S. Moya ${ }^{3}$ \\ ${ }^{1}$ Department of Physics, The Catholic University of America, Washington, D.C. 20064, USA \\ ${ }^{2}$ NASA Goddard Space Flight Center, Greenbelt, MD 20771, USA \\ ${ }^{3}$ Departamento de Fisica, Facultad de Ciencias, Universidad de Chile, Las Palmeras 3425, Casilla 653, Santiago, Chile \\ *visiting: Dept. of Geophysics and Planetary Sciences, Tel Aviv University, Tel Aviv, Israel
}

Received: 24 February 2011 - Revised: 25 April 2011 - Accepted: 3 June 2011 - Published: 20 June 2011

\begin{abstract}
Remote sensing and in-situ observations show that solar wind ions are often hotter than electrons, and the heavy ions flow faster than the protons by up to an Alfvén speed. Turbulent spectrum of Alfvénic fluctuations and shocks were detected in solar wind plasma. Cross-field inhomogeneities in the corona were observed to extend to several tens of solar radii from the Sun. The acceleration and heating of solar wind plasma is studied via 1-D and 2-D hybrid simulations. The models describe the kinetics of protons and heavy ions, and electrons are treated as neutralizing fluid.The expansion of the solar wind is considered in 1-D hybrid model. A spectrum of Alfvénic fluctuations is injected at the computational boundary, produced by differential streaming instability, or initial ion temperature anisotropy, and the parametric dependence of the perpendicular heating of $\mathrm{H}^{+}-\mathrm{He}^{++}$solar wind plasma is studied. It is found that $\mathrm{He}^{++}$ions are heated efficiently by the Alfvénic wave spectrum below the proton gyroperiod.
\end{abstract}

Keywords. Interplanetary physics (Solar wind plasma) Space plasma physics (Kinetic and MHD theory; Numerical simulation studies)

\section{Introduction}

The solar wind plasma plays a major role in the physical connection between the Sun and the Earth. However, despite recent advances in observations and modeling the exact physics of the heating and the acceleration of the solar wind plasma is poorly understood. The spectrum of magnetic fluctuations, composition, and the kinetic properties of protons and ions provide clues on these processes. So far the properties of the solar wind have been observed by remote sensing within $<0.3 \mathrm{AU}$, and measured in-situ from $0.3 \mathrm{AU}$ outward in the

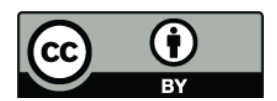

Correspondence to: L. Ofman (leon.ofman@nasa.gov)
Heliosphere. The exact velocity distribution of solar wind protons and heavier ions, and the magnetic field fluctuations close to the Sun in the solar wind acceleration region are not well known. In the future, missions like NASA's Solar Probe+ and ESA's Solar Orbiter will provide measurements inside this distance, that will likely improve our understanding of solar wind acceleration.

The temperature anisotropy of protons deduced from remote sensing observations in coronal holes and in-situ measurements of fast solar wind streams at distances $>0.3 \mathrm{AU}$ provide indirect evidence for the presence of the ioncyclotron waves in coronal plasma, since the anisotropy is a signature of resonant absorption of these waves leading to perpendicular (to the ambient magnetic field) heating. SOHO Ultraviolet Coronagraph Spectrometer (UVCS) observations show that heavy ions such as $\mathrm{O}^{5+}$, and $\mathrm{Mg}^{9+}$ have experienced preferential perpendicular heating, are hotter and flow faster in coronal holes than protons (Kohl et al., 1997; Li et al., 1998; Cranmer et al., 1999). Purely adiabatic expansion of the solar plasma is expected to result in decrease of perpendicular temperate, contrary to observations (e.g. Marsch et al., 1982; Gazis and Lazarus, 1982). Observations of Alfvénic fluctuations in the solar wind by Helios, Ulysses, ACE, and Wind spacecraft show that these fluctuations follow power laws of $f^{-1}$ and $f^{-5 / 3}$ (where $f$ is the frequency), with steeper spectrum at frequencies close to or beyond the proton gyroresonant frequency (Goldstein et al., 1995; Leamon et al., 1998; Podesta et al., 2006; Smith et al., 2006; Vasquez et al., 2007). The above power law indices are typical of self-organized criticality (e.g. Freeman et al., 2000) and turbulence (Kolmogorov, 1941), respectively.

Wave heating of the solar wind was considered in the past, and numerical models of solar wind heating by resonant and nonresonat waves have been developed (see the review by Ofman, 2010b). Theoretical studies of ion-cyclotron resonance have been developed and applied to the heating of the solar corona and the solar wind (Marsch et al., 1982; Axford and McKenzie, 1992; Tu and Marsch, 1997; Li et al.,

Published by Copernicus Publications on behalf of the European Geosciences Union. 
1999; Hollweg, 2000; Hu et al., 2000; Cranmer, 2000; Hollweg and Isenberg, 2002). However, there are theoretical difficulties with the application of the ion-cyclotron mechanism for coronal heating, and its role is not yet fully understood (Ofman and Davila, 1997; Cranmer, 2000; Isenberg, 2004). The limitation of the fluid or linear kinetic models is the assumption of fixed-shape ion velocity distribution and in quasi-linear theory the distribution changes are assumed within quasi-linear limits. In the hybrid models the electrons are treated as a fluid, and the ions are treated fully kinetically as particles, while the self-consistent fields are obtained on a finite grid. Thus, the above limitations are removed in hybrid models allowing fully nonlinear evolution of the ion velocity distributions and the corresponding fields and waves. The results of the models can be compared to observed velocity distributions and magnetic field fluctuations when they become available in the solar wind acceleration region, for example, from the planned NASA's Solar Probe+ mission (McComas et al., 2008).

Hybrid simulations allow relaxing many approximations used in the fluid, multi-fluid, and in linear or quasi-linear kinetic theory and can represent more completely and selfconsistently the wave-particle interactions in the multi-ion solar wind magnetized plasma than previous analytical models. These models have been used successfully to study the nonlinear interactions between a spectrum of waves, beams, and ions in solar wind plasma and the resulting anisotropic heating (Gary et al., 2001, 2003; Ofman et al., 2001, 2002, 2005; Xie et al., 2004; Ofman and Viñas, 2007; Ofman, 2010a). The effects of solar wind expansion of the evolution of the wave-particles interactions and on the heating was also studied (Liewer et al., 2001; Hellinger et al., 2003, 2005; Hellinger and Trávníček, 2008).

Recently, Ofman (2010a) reported the results of parametric studies of 2-D hybrid models of inhomogeneous solar wind plasma heating by a spectrum of Alfvénic fluctuations. The results of that study were motivated by future Solar Probe+ mission that will investigate solar wind proton and $\mathrm{He}^{++}$components near the Sun at about 10 solar radii (McComas et al., 2008). In this region the solar wind plasma density is inhomogeneous due to quasi-steady structures (compared to kinetic time scales) as evident from SOHO/LASCO observations (e.g., DeForest et al., 2001). In Ofman (2010a) study, it was found that the magnetic fluctuations power spectrum steepens in regions of higher density and higher heating rate, while in lower density regions the spectral index is close to $-5 / 3$. The higher heating results due to Alfvén wave refraction by the density inhomogeneity and formation of small scales facilitating dissipation. Here, we describe some recent results of 2-D hybrid models of inhomogeneous solar wind plasma heated by a spectrum of resonant waves, and the results of 1-D hybrid model that considers the effect of expansion on the evolution of the plasma temperature anisotropy and the $\mathrm{p}-\mathrm{He}^{++}$relative drift.

\section{Hybrid model of solar wind plasma}

Hybrid codes represent ions numerically as particles and electrons as a fluid, and so require relatively modest computational resources by comparison with full particle-in-cell codes that represent both ions and electrons as particles, since electron kinetics, spatial scales, and time-scales are neglected (e.g. Winske and Omidi, 1993). The trade-off is that hybrid codes only represent plasma phenomena with frequencies near or below the proton cyclotron frequency. But this is precisely the regime of interest for solar wind acceleration problems where the momentum is carried by the ions. The hybrid model allows one to resolve the ion dynamics and to integrate the equations over many ion-cyclotron periods, while neglecting the small temporal- and spatial scales of the electron kinetic motions. The field equations are solved on a finite grid, using the currents and charge densities produced by the particles in the grid. In a typical calculation the three components of order million particle velocities are used to calculate the currents, and the fields in the 2-D grid. For example, in Ofman and Viñas (2007) study $128 \times 128$ grid with 100 particles/cell/species were used. Note, that each numerical particle represents large number of real particles, determined by the density normalization. The required number of particles per cell is determined by the required limitation on the overall statistical noise. The following equations of motion are solved for each particle of all species $(k)$ :

$$
\begin{aligned}
\frac{d \boldsymbol{x}_{k}}{d t} & =\boldsymbol{v}_{k} \\
m_{k} \frac{d \boldsymbol{v}_{k}}{d t} & =Z_{k} e\left(\boldsymbol{E}+\frac{\boldsymbol{v}_{k} \times \boldsymbol{B}}{c}\right)
\end{aligned}
$$

where $m_{k}$ is the particles mass, $Z_{k}$ is the charge number, $e$ is the electron charge, and $c$ is the speed of light. The electron momentum equation is solved by neglecting the electron inertia

$$
\frac{\partial}{\partial t} n_{\mathrm{e}} m_{\mathrm{e}} \boldsymbol{v}_{\mathrm{e}}=0=-e n_{\mathrm{e}}\left(\boldsymbol{E}+\frac{\boldsymbol{v}_{\mathrm{e}} \times \boldsymbol{B}}{c}\right)-\nabla p_{\mathrm{e}},
$$

where the scalar electron pressure $p_{\mathrm{e}}=k_{B} n_{\mathrm{e}} T_{\mathrm{e}}$ is used for closure, and quasi-neutrality implies $n_{\mathrm{e}}=n_{\mathrm{p}}+Z_{\mathrm{i}} n_{\mathrm{i}}$, where $n_{k}$ is the number density of electron, protons, and ions, respectively, and $T_{\mathrm{e}}$ is the electron temperature. The above equations are supplemented with Maxwell's equations, namely, $\nabla \times \boldsymbol{B}=\frac{4 \pi}{c} \boldsymbol{J}$, and $\nabla \times \boldsymbol{E}=-\frac{1}{c} \frac{\partial \boldsymbol{B}}{\partial t}$ for selfconsistency. The field solutions are obtained on a 2-D grid, and the proton and ion equations of motions are advanced in time as the particle motions respond to the fields at each time step. The method has been tested and used successfully in many studies. The particle and field equations are integrated in time using the Rational Runge-Kutta method (Wambecq, 1978) whereas the spatial derivatives are calculated by pseudospectral FFT method. The hybrid model allows computing the self consistent evolution of the velocity distribution of the ions that includes the nonlinear effects of wave-particle 
interactions without additional assumptions. Moreover, the hybrid model is well suited to describe the nonlinear saturated state of the plasma.

The hybrid code is a robust, well-documented simulation tool which has been used to study various electromagnetic ion instabilities (McKean et al., 1994; Gary et al., 1997; Daughton et al., 1999; Gary et al., 2000, 2001, 2003; Ofman et al., 2001; Xie et al., 2004; Ofman and Viñas, 2007; Ofman, 2010a). Both 1-D and 2-D hybrid codes solve for the full three components of velocity for the ions; the critical difference between the two types of codes is in the dimensionality of the magnetic field fluctuation solver. Comparisons between one- and two-dimensional hybrid simulations often show qualitative agreement in the ion response (Winske and Quest, 1986; Ofman and Viñas, 2007). But 1-D simulation is limited to a discrete number of modes which propagate in a single direction with respect to the background magnetic field, whereas 2-D simulations represent fluctuations propagating across a plane with an arbitrary angle with respect to the background magnetic field, which can more realistically represent observations. In addition 2-D codes allow investigating inhomogeneous background field, and density structure. Thus, waves propagating obliquely to the direction of the background field can be incorporated in the 2-D hybrid model self-consistently.

\subsection{Expanding box model}

The basic equations for the simulation code with expansion have been derived by Grappin and Velli (1996) for an MHD fluid code and Liewer et al. (2001) for a hybrid code. Following Liewer et al. (2001) work, the particle position updates according to Eq. (16) of their paper. The updates for the particle velocities have additional terms, which follow Eq. (17) in that paper. The magnetic field updates follows from Eqs. (5b) and (5f) of Grappin and Velli (1996), but with the flow velocity and density of the ions obtained by moments over the particles of the hybrid simulation. The expanding box equations are obtained with the transformation

$R(t)=R_{0}+U_{0} t$,

where $R(t)$ is the time dependent position of a parcel of plasma in the solar wind propagating from $R_{0}$ at a constant velocity $U_{0}$. The dimensionless quantity

$a(t)=R(t) / R_{0}=1+\frac{U_{0}}{R_{0}} t$,

and a Galilean transformation $v^{\prime}=v-U_{0}$. Assuming "slow" expansion we define the small quantity $\epsilon=\frac{U_{0}}{R_{0}} t \ll 1$, neglecting terms of $O\left(\epsilon^{2}\right)$. Thus the coordinates are transformed to a moving frame as follows:

$x=x^{\prime}+R, y=a y^{\prime}, z=a z^{\prime}$.

Note, that the $\mathrm{x}$-coordinate undergoes Galilean transformation, while the $\mathrm{y}$ - and $\mathrm{z}$-coordinates undergo stretching. Using the above transformation we can write the time and space derivatives in terms of the primed coordinates and transform the equations to be solved in the hybrid code

$\frac{d \mathbf{v}}{d t}=\frac{d \mathbf{v}^{\prime}}{d t^{\prime}}+\frac{U_{0}}{R_{0}} \mathbf{P} \cdot \mathbf{v}^{\prime}$,

where

$\mathbf{P}=\left(\begin{array}{lll}0 & 0 & 0 \\ 0 & 1 & 0 \\ 0 & 0 & 1\end{array}\right)$,

and where to calculate $\mathbf{v}^{\prime}$ we have used

$\frac{d x^{\prime}}{d t}=v_{x}^{\prime}, \frac{d y^{\prime}}{d t}=\frac{1}{a} v_{y}^{\prime}, \frac{d z^{\prime}}{d t}=\frac{1}{a} v_{z}^{\prime}$,

and for the magnetic field

$$
\begin{aligned}
\frac{\partial \mathbf{B}^{\prime}}{\partial t^{\prime}} & +\mathbf{B}^{\prime}\left(\nabla^{\prime} \cdot \mathbf{U}_{i}^{\prime}\right)-\left(\mathbf{B}^{\prime} \cdot \nabla^{\prime}\right) \mathbf{U}_{i}^{\prime}+\left(\mathbf{U}_{i}^{\prime} \cdot \nabla^{\prime}\right) \mathbf{B}^{\prime} \\
& +\frac{c}{4 \pi e n_{e}} \nabla^{\prime} \times\left[\left(\nabla^{\prime} \times \mathbf{B}^{\prime}\right) \times \mathbf{B}^{\prime}\right]= \\
& =-\frac{2 U_{0}}{R_{0}} B_{x}^{\prime} \hat{x}-\frac{U_{0}}{R_{0}} B_{y}^{\prime} \hat{y}-\frac{U_{0}}{R_{0}} B_{z}^{\prime} \hat{z}
\end{aligned}
$$

where the transformed ion bulk velocity is $\mathbf{U}_{i}^{\prime}=\mathbf{U}_{i}-\mathbf{U}_{0}$, and the electric field in the moving frame is

$$
\begin{aligned}
\mathbf{E}^{\prime}= & -\frac{1}{c} \mathbf{U}_{i}^{\prime} \times \mathbf{B}^{\prime}+\frac{1}{4 \pi e n_{e}}\left(\nabla^{\prime} \times \mathbf{B}^{\prime}\right) \times \mathbf{B}^{\prime} \\
& -\frac{1}{e n} \nabla^{\prime}\left(n_{e} k_{B} T_{e}\right),
\end{aligned}
$$

Note, that by using vector potential $\boldsymbol{A}$, where $\mathbf{B}=\nabla \times \boldsymbol{A}$, Eq. (20) of Liewer et al. (2001) is recovered. The above equations are implemented in our 1-D hybrid code. In this code, all three components of velocities and fields are maintained, but the derivatives with respect to $y^{\prime}$ and $z^{\prime}$ are zero.

\section{Numerical results}

Detailed parametric studies of inhomogeneous plasma heating are described in recent study by Ofman (2010a). The results of 2-D hybrid model of inhomogeneous plasma heating for a different (Gaussian) inhomogeneous background are described in Sect. 3.1. The first results with our 1-D expanding box hybrid model are presented in Sect. 3.2.

\subsection{Inhomogeneous plasma results}

Motivated by planned missions to the inner heliosphere, such as the Solar Probe+ (McComas et al., 2008) we study the effect of solar wind density inhomogeneity across the magnetic field. Observations show (e.g., DeForest et al., 2001) that at about 10 solar radii (the perihelion of Solar Probe+) there are quasi-steady density structures extending from the solar corona, persistent on time scales much longer than considered here. These structures can affect the dissipation of the 

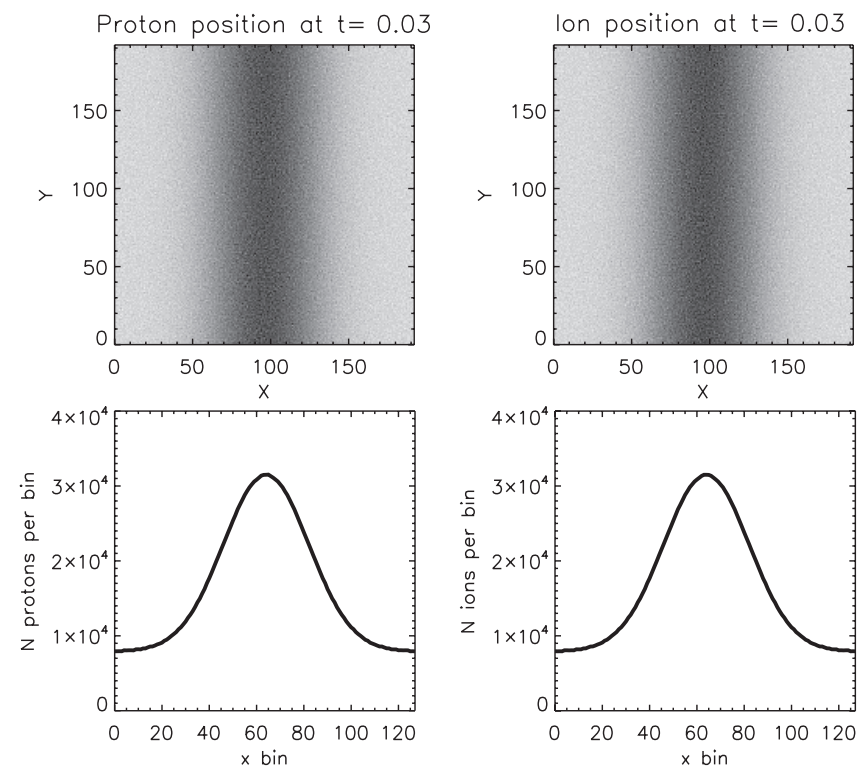

Fig. 1. The initial spatial distributions of protons (left panel) and $\mathrm{He}^{++}$ions (right panel) in the inhomogeneous 2-D hybrid model.

Alfvénic fluctuations propagating in the solar wind. In order to study the effects of an inhomogeneous proton and $\mathrm{He}^{++}$ ion density the 2-D hybrid model computations are initialize with the following expression in (see, Ofman, 2010a):

$n_{k}(x)=n_{0, k}\left[1+n_{0 m} e^{-\left(\frac{x-x_{0}}{w}\right)^{q}}\right]$,

where $x_{0}$ is the location of the central symmetry axis, $n_{0 m}$ is the amplitude of the inhomogeneity (in Ofman, 2010a, study the values of $n_{0 m}$ were in the range $0-4$, where $n_{0 m}=0$ corresponds to homogeneous plasma; here we use $\left.n_{0 m}=2\right), w=38.4 \Delta$ determines the width of the high density region, where $\Delta=c / \omega_{p p}$ is the proton inertia length, and $\omega_{p p}=\left(4 \pi n_{p} e^{2} / m_{p}\right)^{1 / 2}$ is the proton plasma frequency, $k$ is the index for protons, and $\mathrm{He}^{++}$ions, and $q$ is the power that determines the sharpness of the transition between the low and high density region. As in Ofman (2010a) the size of the grid is $192 \Delta$ in each direction, and the resolution is $128 \times 128$ with 127 particles per cell of each species. In Ofman (2010a) the value $q=6$ was used, and detailed description of the boundary conditions, the method for the generation of the driving Alfvénic fluctuations, and the method of solution with the numerical parameters are described. Here, we use the same method to study the effects of less-sharp Gaussian inhomogeneity $(q=2)$ on plasma heating. The two-dimensional spatial distribution of the proton and $\mathrm{He}^{++}$ ions density and the corresponding Gaussian density profile are shown in Fig. 1. The protons number density is 0.9 and the $\mathrm{He}^{++}$ion density is 0.05 in units of electron density. The electron thermal to magnetic pressure ratio $\beta_{e}=0.02$, and the initial proton and ion $\beta_{p}=\beta_{i}=0.04$.
A spectrum of circularly-polarized Alfvénic fluctuations with amplitude $B_{z 0}=0.04$ and $f^{-1}$ frequency dependence in a frequency band below the proton gyrofrequency $\Omega_{p}=\frac{e B}{m_{p} c}$ was driven continuously at $y=0$ boundary. In the model the spectrum spans frequencies below the protons gyroresonat frequency but includes the $\mathrm{He}^{++}$ion resonant frequency that is $0.5 \Omega_{p}$. From linear theory (e.g. Gary, 1993), the resonance condition for left-hand polarized parallel propagating ion-cyclotron waves is $\omega-k_{\|} v_{\|, i}=\Omega_{i}$, where $\omega$ is the resonant frequency, $k_{\|}$is the parallel (to the background magnetic field) wavenumber, $v_{\|, i}$ is the ion parallel velocity, and $\Omega_{i}=\frac{Z e B}{A m_{p} c}$ is the ion gyrofrequency, where $Z$ is the charge number, and $A$ is the mass number of the ions. Therefore, the alpha particle distribution resonate with the input fluctuations spectrum, while the non-resonant protons are not heated. There is no initial drift between the protons and $\mathrm{He}^{++}$ions in this case. As a result, the plasma is heated in three stages in a nonlinear process (see Fig. 2): first, the $\mathrm{He}^{++}$ions are heated by absorbing the resonant left hand polarized part of the frequency fluctuations spectrum. This leads to the increase of $\mathrm{He}^{++}$ion perpendicular temperature and anisotropy to $\sim 7$. As a result, the $\mathrm{He}^{++}$ion distribution becomes unstable to ion-cyclotron instability (e.g., Gary et al., 2001, 2003) and emit a spectrum of magnetic fluctuations while relaxing the anisotropy of $\mathrm{He}^{++}$temperature. The left hand circularly polarized part of the emitted spectrum that matches the proton resonant condition leads to proton heating. This results in increase of proton temperature anisotropy to $\sim 1.2$. Finally, both, protons and $\mathrm{He}^{++}$ions are heated in the parallel direction due to velocity-space diffusion, resulting in decrease of the temperature anisotropy of both particle species.

The inclusion of background density inhomogeneity leads to the refraction of Alfvénic fluctuations due to nonuniformity of the local Alfvén speed. The refraction facilitates the formation of small scales in the inhomogeneous and the higher density (lower Alfvén speed) regions. As a result, the dissipation of the fluctuations and the heating of the plasma is more efficient than in the homogeneous case. This effect was demonstrated and investigated in detail in a parametric study by Ofman (2010a). Here, by comparing the results of the inhomogeneous case to the results of the homogeneous plasma (see Fig. 2) it is evident that the heating is more efficient in Gaussian inhomogeneous profile then in the homogeneous case. Parallel and perpendicular heating, as well as the anisotropy of protons and $\mathrm{He}^{++}$ions increases to larger valued in the inhomogeneous case compared to homogeneous case.

The perpendicular velocity distribution of $\mathrm{He}^{++}$ions is shown at $t=450 \Omega_{p}^{-1}$ in Fig. 3 . The cut through the distribution at $V_{\mathrm{z}}=0$ is shown in the lower panel of the figure. The best-fit Maxwellian velocity distribution is shown (dashes). It is evident that the distribution is close to Maxwellian in the core, with non-thermal tails at higher velocities. The tails 

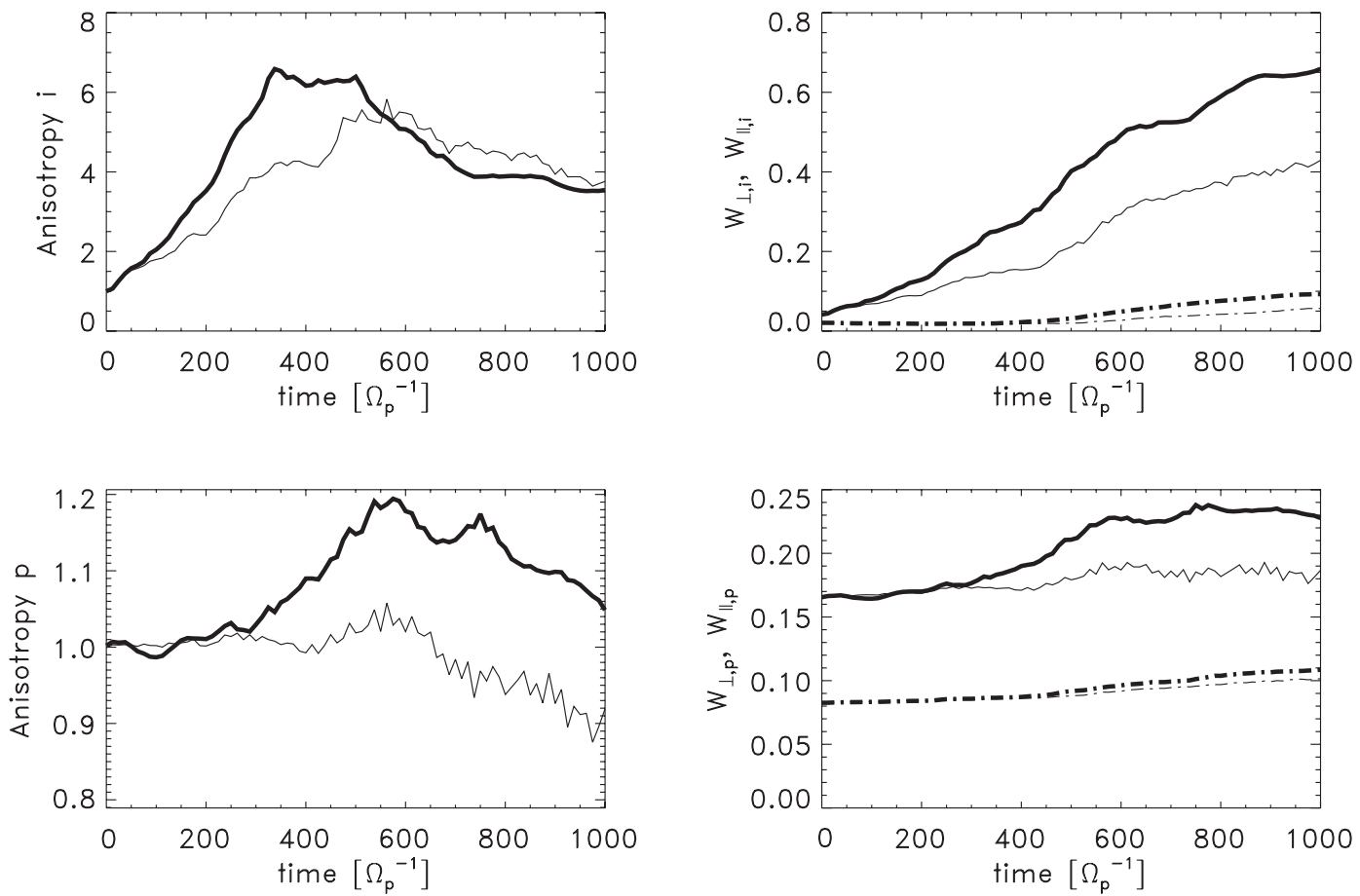

Fig. 2. The left panels show the evolution of the anisotropy of $\mathrm{He}^{++}$ions (top panel) and protons (lower panel). The right panels show the corresponding evolution of the perpendicular (solid) and parallel (dot-dashes) kinetic energies of $\mathrm{He}^{++}$ions and protons for the 2-D hybrid model of plasma heating with Alfvénic fluctuations spectrum. The thick curves are for the inhomogeneous plasma, Eq. (12), and the thin curves are for homogeneous background density.

are the results of non-equilibrium heating process, and their magnitude decreases at later times $\left(\sim 1000 \Omega_{p}^{-1}\right.$, not shown $)$ as new equilibrium state is approached.

\subsection{Expanding box model results}

The linear (Vlasov) stability theory of anisotropic or drifting plasma populations was discussed extensively in the past (Gary, 1993, e.g.). The validity and applicability of the linear stability analysis was tested with 1-D and 2-D hybrid models of two-ion plasma (Ofman et al., 2001, 2002; Gary et al., 2001, 2003; Xie et al., 2004; Ofman and Viñas, 2007). It was found that the threshold condition for instability of drifting ion-proton plasma is the requirement that the drift velocity is larger than the local Alfvén speed. The threshold of ion-cyclotron instability of anisotropic population of ions obtained from linear dispersion relation and verified by hybrid models is that the temperature anisotropy $T_{\perp, i} / T_{\|, i}>S\left(\beta_{\|, i}\right)$ exceeds a parameter dependent threshold, $S\left(\beta_{\|, i}\right)>1$, determined by the ratio of the parallel thermal and magnetic pressure, $\beta_{\|, i}$ (e.g. Gary and Lee, 1994; Ofman et al., 2001; Gary et al., 2001; Xie et al., 2004). In this section we present the results of expanding box 1-D hybrid model of homogeneous plasma for two cases: (1) initially anisotropic unstable $\mathrm{He}^{++}$ion distribution, with isotropic proton distribution, and without drift; (2) initially isotropic proton and $\mathrm{He}^{++}$ion distribution with super-Alfvénic drift.The above initial states (calculated separately) are used as the sources of free energy that produce the ion-cyclotron instability and waves in the models. We compare the results of the two cases without expansion to the expanding cases, thus, demonstrating the effects of expansion on the evolution of the plasma heating.

The effect of solar wind expansion on the relaxation of the temperature anisotropy, $T_{k, \perp} / T_{k, \|}$, of protons and $\mathrm{He}^{++}$ ions in the three component plasma was studied. For this purpose the 1-D hybrid expanding-box code was run with initially isotropic protons $(92 \%)$ and anisotropic $\mathrm{He}^{++}$ions (4\%) with $T_{\mathrm{i}, \perp} / T_{\mathrm{i}, \|}=10$ at $t=0$. In this model the initial $\beta_{\mathrm{e}}=\beta_{\mathrm{p}}=\beta_{\mathrm{i}}=0.05$, and the field solutions were obtained in 1024 grid cells with cell size $0.125 \Delta$, and with 160 particles per cell of each ion species. Two cases are compared to non-expanding solutions $(\epsilon=0): \epsilon=10^{-3}$, and $\epsilon=2 \times 10^{-3}$. As in Liewer et al. (2001) the expansion rate is about two orders of magnitude faster then the solar wind expansion rate so that the evolution takes place on a short time scale due to numerical limitations. In Fig. 4 the temporal evolution of the temperature anisotropy $T_{k, \perp} / T_{k, \|}$ is shown. It is evident that with expansions, the $\mathrm{He}^{++}$ion temperature anisotropy relaxes more rapidly than without expansion. The proton temperature also become anisotropic due to expansion, with $T_{\mathrm{p}, \perp} / T_{\mathrm{p}, \|}<1$. Further decrease in $T_{\mathrm{p}, \perp} / T_{\mathrm{p}, \|}$ can lead to fire-hose instability at later times when 

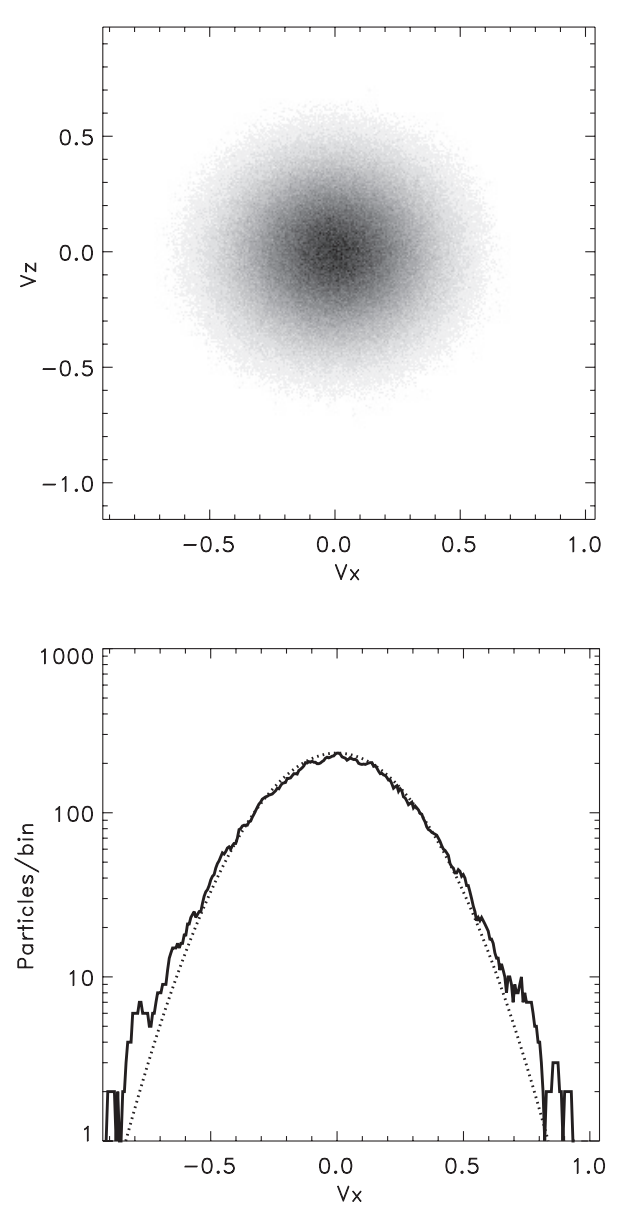

Fig. 3. The perpendicular velocity distribution of $\mathrm{He}^{++}$ions (top) and the corresponding cut of the distribution at $V_{\mathrm{Z}}=0$ showing best fit Maxwellian distribution (dashes). The velocity is in units of the Alfvén speed.

the threshold for marginal stability is reached (e.g Gary et al., 1998). In the present study the solutions did not reach this threshold in the modeled time $\left(200 \Omega_{\mathrm{p}}^{-1}\right)$. Note, that the $\mathrm{He}^{++}$parallel temperature increases and perpendicular temperature decreases rapidly leading to anisotropy decrease to $\sim 2$ within $\sim 100 \Omega_{\mathrm{p}}^{-1}$ consistent with the ion-cyclotron instability marginally stable value (Gary et al., 2001). It is evident that when expansion is present the $\mathrm{He}^{++}$ion temperature anisotropy continues to decrease gradually, below the ion-cyclotron marginally stable value.

The effect of solar wind expansion is demonstrated on an initially drifting populations of $\mathrm{p}-\mathrm{He}^{++}$ions in Fig. 5. The initial drift velocity was $U_{\mathrm{x}, \text { drift }}=U_{\mathrm{x}, \mathrm{p}}-U_{\mathrm{x}, \mathrm{i}}=2$ in units of the Alfvén speed, above the threshold for beam instability (as discussed above). As a result of the instability the magnetic fluctuations increase rapidly (within $\sim 100 \Omega_{\mathrm{p}}^{-1}$ ) leading to perpendicular heating of $\mathrm{He}^{++}$ions to the marginally stable temperature anisotropy of $\sim 2$. Consequently, the drift velocity has decreased transferring the kinetic energy to (mostly)

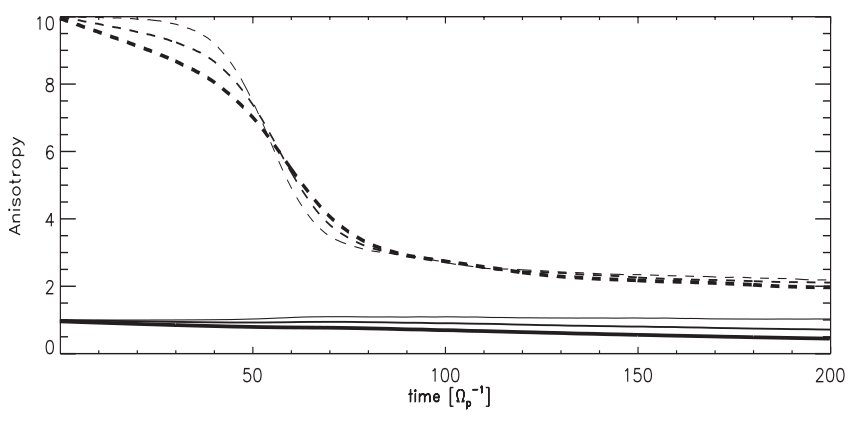

Fig. 4. The temporal evolution of the temperature anisotropy for protons (solid) and $\mathrm{He}^{++}$ions (dashes) obtained with the 1-D expanding box hybrid model for initially anisotropic $\mathrm{He}^{++}$temperature. The thin curves show the temporal evolution without expansion $(\epsilon=0)$, the thicker curves are for $\epsilon=10^{-3}$, and the thick curves are for $\epsilon=2 \times 10^{-3}$.

perpendicular heating. The effect of the expansion on this process is evident by comparing the results for $\epsilon=0,10^{-3}$, $2 \times 10^{-3}$. The solar wind expansion leads to the decrease of proton and $\mathrm{He}^{++}$perpendicular temperatures, and consequently to the decrease of the anisotropy. The magnetic fluctuations increase somewhat, and the drift decreases to a lower level when expansion is present.

\section{Conclusions}

The heating of the solar wind plasma by Alfvénic fluctuations spectrum is investigated with 1-D and 2-D hybrid models. The effects of inhomogeneity on the heating is considered in a 2-D hybrid model. Here, the Gaussian density inhomogeneity is considered, extending previous studies. In previous studies it was shown that inhomogeneity leads to more efficient plasma heating compared to the homogeneous case due to refraction of the input Alfvén waves in the inhomogeneous layer and formation of small scales, facilitating more efficient heating than in the homogeneous case. Here, this is confirmed further using the Gaussian inhomogeneous density profile. It is found that the plasma heating by a spectrum of Alfvénic fluctuations occurs in three nonlinear stages. First, the $\mathrm{He}^{++}$ions are heated by the resonating left-hand circularly polarized waves increasing their perpendicular temperature, next the protons are heated by the waves produced by the relaxation of the $\mathrm{He}^{++}$temperature anisotropy through ion cyclotron instability and resonance with the waves emitted by the $\mathrm{He}^{++}$ion population. Finally, parallel heating occurs in both ion species due to velocity space diffusion resulting in the decrease of their anisotropy. The 2-D hybrid model results demonstrate the three stage plasma heating, that can take place when the magnetic fluctuations spectrum has most of its power below the proton gyroresonance. This scenario is likely to occur in solar wind plasma, since observation indicate that the solar wind 

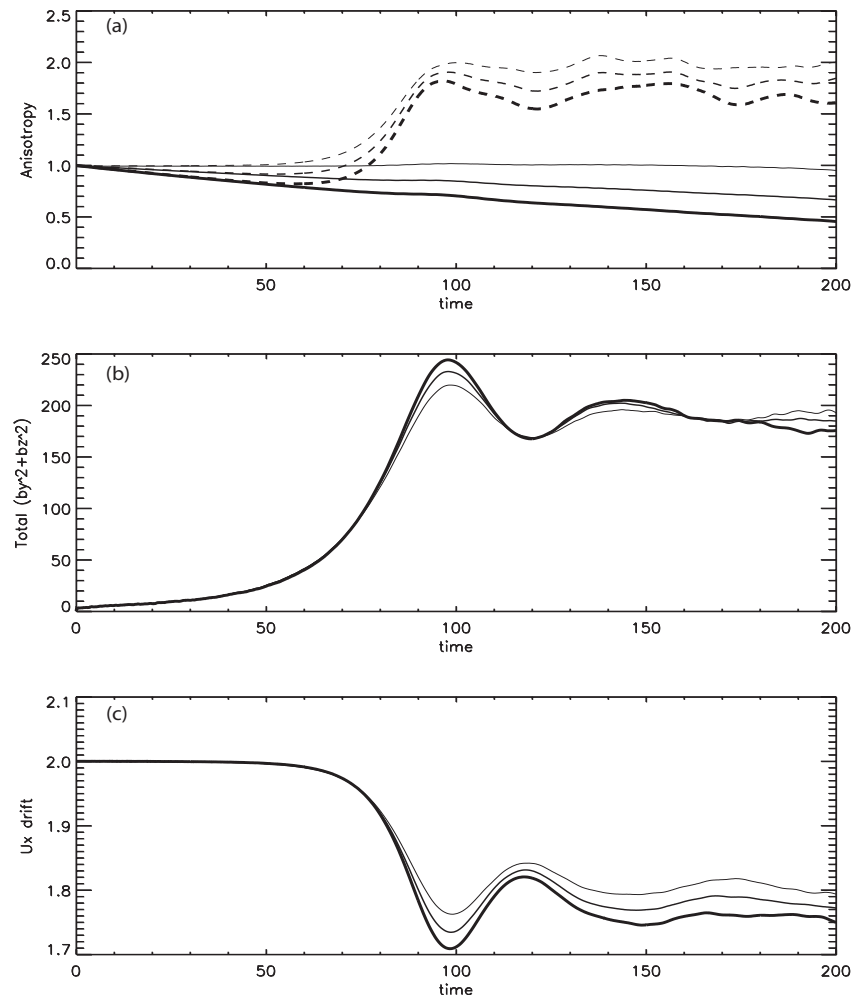

Fig. 5. The temporal evolution of the temperature anisotropy for protons (solid) and $\mathrm{He}^{++}$ions (dashes) obtained with the 1-D expanding box hybrid model for an initially drifting $\mathrm{p}-\mathrm{He}^{++}$population with $U_{\mathrm{x}, \mathrm{drift}}=2$ (in units of the Alfvén speed). (a) The temperature anisotropies. (b) The magnetic fluctuations (arbitrary units). (c) The $\mathrm{p}-\mathrm{He}^{++}$drift velocity. The thin curves show the temporal evolution without expansion $(\epsilon=0)$, the thicker curves are for $\epsilon=10^{-3}$, and the thick curves are for $\epsilon=2 \times 10^{-3}$. The time is in units of $\Omega_{p}^{-1}$.

magnetic fluctuations have most of their power in frequencies below the proton gyroresonant frequency (e.g. Smith et al., 2006). Note, that in the 2-D hybrid model the scattering rate of the ions is somewhat faster than in the 1-D model due to the additional degree of freedom allowing oblique wave propagation.

The solar solar wind expansion is implemented in 1-D hybrid model and the expanding-box equations are solved for two heating scenarios in homogeneous plasma. In the first case effect of solar wind expansion is considered on initially unstable $\mathrm{He}^{++}$population with temperature anisotropy above the stability threshold. In the second case (calculated separately), the effect of expansion is considered on a drifting $\mathrm{p}-\mathrm{He}^{++}$plasma population with initial super-Alfvénic relative drift above the stability threshold. It is found in both cases that the expansion contributes to perpendicular cooling of the ions, and to faster relaxation of the initial $\mathrm{He}^{++}$ anisotropy compared to non-expanding plasma. This is evident in Figs. 4-5 by comparing the expanding and non ex- panding evolution. The relaxation of the drift instability occurs somewhat faster and the decrease of the drift velocity is more pronounced in an expanding solar wind plasma compared to the non-expanding case. The perpendicular cooling of protons can lead eventually to fire-hose instability at much later times (left for a future study) when the perpendicular temperature falls significant below the parallel temperature and threshold for instability is reached (see, e.g. Gary et al., 1998; Hellinger and Trávníček, 2008). Since in-situ observations at $0.3-1 \mathrm{AU}$ show that usually $T_{\mathrm{i} \perp}>T_{\mathrm{i} \|}$ (e.g. Marsch, 1991) strongly suggest that perpendicular heating of the protons and ions takes place continuously, countering the effects of expansion as the solar wind plasma propagates into the heliosphere.

Acknowledgements. LO would like to acknowledge support by NASA grants NNX08AV88G and NNX10AC56G. AFV would like to acknowledge support by NASA grant NNX10AC56G. PSM is grateful to Comisión Nacional de Ciencia y Tecnología (CONICyT, Chile) Doctoral Fellowship D-21070397 and CONICyT/BecasChile fellowship for doctoral internships at NASA/GSFC at 2010. We acknowledge the use of computer resources at NASA's Ames Research Center advanced supercomputing facility.

Guest Editor M. Gedalin thanks S. P. Gary and M. Ruderman for their help in evaluating this paper.

\section{References}

Axford, W. I. and McKenzie, J. F.: The origin of high speed solar wind streams, in: Solar Wind Seven Colloquium, pp. 1-5, 1992.

Cranmer, S. R.: Ion Cyclotron Wave Dissipation in the Solar Corona: The Summed Effect of More than 2000 Ion Species, Astrophys. J., 532, 1197-1208, 2000.

Cranmer, S. R., Field, G. B., and Kohl, J. L.: Spectroscopic Constraints on Models of Ion Cyclotron Resonance Heating in the Polar Solar Corona and High-Speed Solar Wind, Astrophys. J., 518, 937-947, 1999.

Daughton, W., Gary, S. P., and Winske, D.: Electromagnetic proton/proton instabilities in the solar wind: Simulations, J. Geophys. Res., 104, 4657-4668, doi:10.1029/1998JA900105, 1999.

DeForest, C. E., Plunkett, S. P., and Andrews, M. D.: Observation of Polar Plumes at High Solar Altitudes, Astrophys. J., 546, 569575, doi:10.1086/318221, 2001.

Freeman, M. P., Watkins, N. W., and Riley, D. J.: Power law distributions of burst duration and interburst interval in the solar wind: Turbulence or dissipative self-organized criticality?, Physical Review E, 62, 8794-8797, doi:10.1103/PhysRevE.62.8794, 2000.

Gary, S. P.: Theory of Space Plasma Microinstabilities, Cambridge University Press, New York, NY, 1993.

Gary, S. P. and Lee, M. A.: The ion cyclotron anisotropy instability and the inverse correlation between proton anisotropy and proton beta, J. Geophys. Res., 99, 11297-11302, doi:10.1029/94JA00253, 1994.

Gary, S. P., Wang, J., Winske, D., and Fuselier, S. A.: Proton temperature anisotropy upper bound, J. Geophys. Res., 102, 2715927170, doi:10.1029/97JA01726, 1997. 
Gary, S. P., Li, H., O'Rourke, S., and Winske, D.: Proton resonant firehose instability: Temperature anisotropy and fluctuating field constraints, J. Geophys. Res., 103, 14567-14574, doi:10.1029/98JA01174, 1998.

Gary, S. P., Yin, L., Winske, D., and Reisenfeld, D. B.: Electromagnetic alpha/proton instabilities in the solar wind, Geophys. Res. Lett., 27, 1355-1358, 2000.

Gary, S. P., Yin, L., Winske, D., and Ofman, L.: Electromagnetic heavy ion cyclotron instability: Anisotropy constraint in the solar corona, J. Geophys. Res., 106, 10715-10722, doi:10.1029/2000JA000406, 2001.

Gary, S. P., Yin, L., Winske, D., Ofman, L., Goldstein, B. E., and Neugebauer, M.: Consequences of proton and alpha anisotropies in the solar wind: Hybrid simulations, J. Geophys. Res., 108, 1068, doi:10.1029/2002JA009654, 2003.

Gazis, P. R. and Lazarus, A. J.: Voyager observations of solar wind proton temperature - 1-10 AU, Geophys. Res. Lett., 9, 431-434, 1982.

Goldstein, B. E., Smith, E. J., Balogh, A., Horbury, T. S., Goldstein, M. L., and Roberts, D. A.: Properties of magnetohydrodynamic turbulence in the solar wind as observed by Ulysses at high heliographic latitudes, Geophys. Res. Lett., 22, 3393-3396, 1995.

Grappin, R. and Velli, M.: Waves and streams in the expanding solar wind, J. Geophys. Res., 101, 425-444, doi:10.1029/95JA02147, 1996.

Hellinger, P. and Trávníček, P. M.: Oblique proton fire hose instability in the expanding solar wind: Hybrid simulations, J. Geophys. Res., 113, 10109, doi:10.1029/2008JA013416, 2008.

Hellinger, P., Trávníček, P., Mangeney, A., and Grappin, R.: Hybrid simulations of the expanding solar wind: Temperatures and drift velocities, Geophys. Res. Lett., 30, 050000-1, doi:10.1029/2002GL016409, 2003.

Hellinger, P., Velli, M., Trávníček, P., Gary, S. P., Goldstein, B. E., and Liewer, P. C.: Alfvén wave heating of heavy ions in the expanding solar wind: Hybrid simulations, J. Geophys. Res., 110, 12109, doi:10.1029/2005JA011244, 2005.

Hollweg, J. V.: Cyclotron resonance in coronal holes: 3. A fivebeam turbulence-driven model, J. Geophys. Res., 105, 1569915714, doi:10.1029/1999JA000449, 2000.

Hollweg, J. V. and Isenberg, P. A.: Generation of the fast solar wind: A review with emphasis on the resonant cyclotron interaction, J. Geophys. Res., 107, 1147, doi:10.1029/2001JA000270, 2002.

Hu, Y. Q., Esser, R., and Habbal, S. R.: A four-fluid turbulencedriven solar wind model for preferential acceleration and heating of heavy ions, J. Geophys. Res., 105, 5093-5112, doi:10.1029/1999JA900430, 2000.

Isenberg, P. A.: The kinetic shell model of coronal heating and acceleration by ion cyclotron waves: 3. The proton halo and dispersive waves, J. Geophys. Res., 109, A03101, doi:10.1029/2002JA009449, 2004.

Kohl, J. L., Noci, G., Antonucci, E., Tondello, G., Huber, M. C. E., Gardner, L. D., Nicolosi, P., Strachan, L., Fineschi, S., Raymond, J. C., Romoli, M., Spadaro, D., Panasyuk, A., Siegmund, O. H. W., Benna, C., Ciaravella, A., Cranmer, S. R., Giordano, S., Karovska, M., Martin, R., Michels, J., Modigliani, A., Naletto, G., Pernechele, C., Poletto, G., and Smith, P. L.: First Results from the SOHO Ultraviolet Coronagraph Spectrometer, Solar Phys., 175, 613-644, 1997.

Kolmogorov, A.: The Local Structure of Turbulence in Incom- pressible Viscous Fluid for Very Large Reynolds' Numbers, Akademiia Nauk SSSR Doklady, 30, 301-305, 1941.

Leamon, R. J., Matthaeus, W. H., Smith, C. W., and Wong, H. K.: Contribution of Cyclotron-resonant Damping to Kinetic Dissipation of Interplanetary Turbulence, Astrophys. J. Lett., 507, L181L184, doi:10.1086/311698, 1998.

Li, X., Habbal, S. R., Kohl, J., and Noci, G.: The Effect of Temperature Anisotropy on Observations of Doppler Dimming and Pumping in the Inner Corona, Astrophys. J., 501, L133-L137, 1998.

Li, X., Habbal, S. R., Hollweg, J. V., and Esser, R.: Heating and cooling of protons by turbulence-driven ion cyclotron waves in the fast solar wind, J. Geophys. Res., 104, 2521-2536, doi:10.1029/1998JA900126, 1999.

Liewer, P. C., Velli, M., and Goldstein, B. E.: Alfvén wave propagation and ion cyclotron interactions in the expanding solar wind: One-dimensional hybrid simulations, J. Geophys. Res., 106, 29261-29282, doi:10.1029/2001JA000086, 2001.

Marsch, E.: Kinetic Physics of the Solar Wind Plasma, pp. 45-133, Physics of the Inner Heliosphere II. Particles, Waves and Turbulence, XI, 352 pp. 152 figs, Springer-Verlag Berlin Heidelberg New York, also: Physics and Chemistry in Space, volume 21, 2 , p. 45-133, 1991.

Marsch, E., Schwenn, R., Rosenbauer, H., Muehlhaeuser, K.-H., Pilipp, W., and Neubauer, F. M.: Solar wind protons - Threedimensional velocity distributions and derived plasma parameters measured between 0.3 and 1 AU, J. Geophys. Res., 87, 5272, 1982.

McComas, D. J., Acton, L. W., Balat-Pichelin, M., Bothmer, V., Dirling, R. B., Feldman, W. C., Gloeckler, G., Habbal, S. R., Hassler, D. M., Mann, I., Matthaeus, W. H., McNutt, R. L., Mewaldt, R. A., Murphy, N., Ofman, L., Sittler, E. C., Smith, C. W., Velli, M., and Zurbuchen, T. H.: Solar Probe Plus: Report of the Science and Technology Definition Team (STDT), NASA/TM-2008-214161, 2008.

McKean, M. E., Winske, D., and Gary, S. P.: Two-dimensional simulations of ion anisotropy instabilities in the magnetosheath, J. Geophys. Res., 99, 11141-11154, doi:10.1029/93JA03025, 1994.

Ofman, L.: Hybrid model of inhomogeneous solar wind plasma heating by Alfvén wave spectrum: Parametric studies, J. Geophys. Res., 115, 4108, doi:10.1029/2009JA015094, 2010a.

Ofman, L.: Wave Modeling of the Solar Wind, Living Reviews in Solar Physics, 7, 4, $2010 \mathrm{~b}$.

Ofman, L. and Davila, J. M.: Do First Results from SOHO UVCS Indicate That the Solar Wind Is Accelerated by Solitary Waves?, Astrophys. J., 476, L51-L54, 1997.

Ofman, L. and Viñas, A. F.: Two-dimensional hybrid model of wave and beam heating of multi-ion solar wind plasma, J. Geophys. Res., 112, 6104, doi:10.1029/2006JA012187, 2007.

Ofman, L., Viñas, A., and Gary, S. P.: Constraints on the $\mathrm{O}^{+5}$ Anisotropy in the Solar Corona, Astrophys. J. Lett., 547, L175L178, doi:10.1086/318900, 2001.

Ofman, L., Gary, S. P., and Viñas, A.: Resonant heating and acceleration of ions in coronal holes driven by cyclotron resonant spectra, J. Geophys. Res., 107, 1461-1469, 2002.

Ofman, L., Davila, J. M., Nakariakov, V. M., and Viñas, A.F.: High-frequency Alfvén waves in multi-ion coronal plasma: Observational implications, J. Geophys. Res., 110, 9102, 
doi:10.1029/2004JA010969, 2005.

Podesta, J. J., Roberts, D. A., and Goldstein, M. L.: Power spectrum of small-scale turbulent velocity fluctuations in the solar wind, J. Geophys. Res., 111, 10109, doi:10.1029/2006JA011834, 2006.

Smith, C. W., Hamilton, K., Vasquez, B. J., and Leamon, R. J.: Dependence of the Dissipation Range Spectrum of Interplanetary Magnetic Fluctuationson the Rate of Energy Cascade, Astrophys. J. Lett., 645, L85-L88, doi:10.1086/506151, 2006.

Tu, C.-Y. and Marsch, E.: Two-Fluid Model for Heating of the Solar Corona and Acceleration of the Solar Wind by High-Frequency Alfven Waves, Solar Phys., 171, 363-391, 1997.

Vasquez, B. J., Smith, C. W., Hamilton, K., MacBride, B. T., and Leamon, R. J.: Evaluation of the turbulent energy cascade rates from the upper inertial range in the solar wind at $1 \mathrm{AU}, \mathrm{J}$. Geophys. Res., 112, 7101, doi:10.1029/2007JA012305, 2007.
Wambecq, A.: Rational Runge-Kutta Methods for Solving Systems of Ordinary Differential Equations, Computing, 20, 333-342, 1978.

Winske, D. and Omidi, N.: Computer Space Plasma Physics: Simulation Techniques and Sowftware, edited by: Matsumoto, H. and Omura, Y., Terra Scientific Publishing, Tokyo, 1993.

Winske, D. and Quest, K. B.: Electromagnetic ion beam instabilities - Comparison of oneand two-dimensional simulations, J. Geophys. Res., 91, 8789-8797, 1986.

Xie, H., Ofman, L., and Viñas, A.: Multiple ions resonant heating and acceleration by Alfvén/cyclotron fluctuations in the corona and the solar wind, J. Geophys. Res., 109, A08103, doi:10.1029/2004JA010501, 2004. 